\title{
Multifocal posterior uveitis: clinical and pathological findings
}

\author{
David G Charteris, William R Lee
}

\begin{abstract}
A pathological study was performed on the necropsy eyes of a 59-year old-woman who had suffered for nine years from multifocal posterior uveitis. The disease had been controlled by steroid therapy with good preservation of visual function. Extensive investigation did not reveal the aetiology. On macroscopic examination numerous focal lesions with various degrees of pigmentation were observed scattered across the fundi. These lesions were studied by light and electron microscopy and immunohistochemistry. There was ongoing chorioretinal inflammation in the foci, producing destruction of Bruch's membrane, the retinal pigment epithelium (RPE), and the outer retina. The focal scars showed migration of RPE and glial cells and neovascularisation. Capillary and venule endothelial cells were swollen at the inflammatory sites. Attempts to establish a cause for this condition were unsuccessful.
\end{abstract}

The pathogenesis of many forms of multifocal chorioretinal inflammatory disease remains unknown, and a lack of histopathological

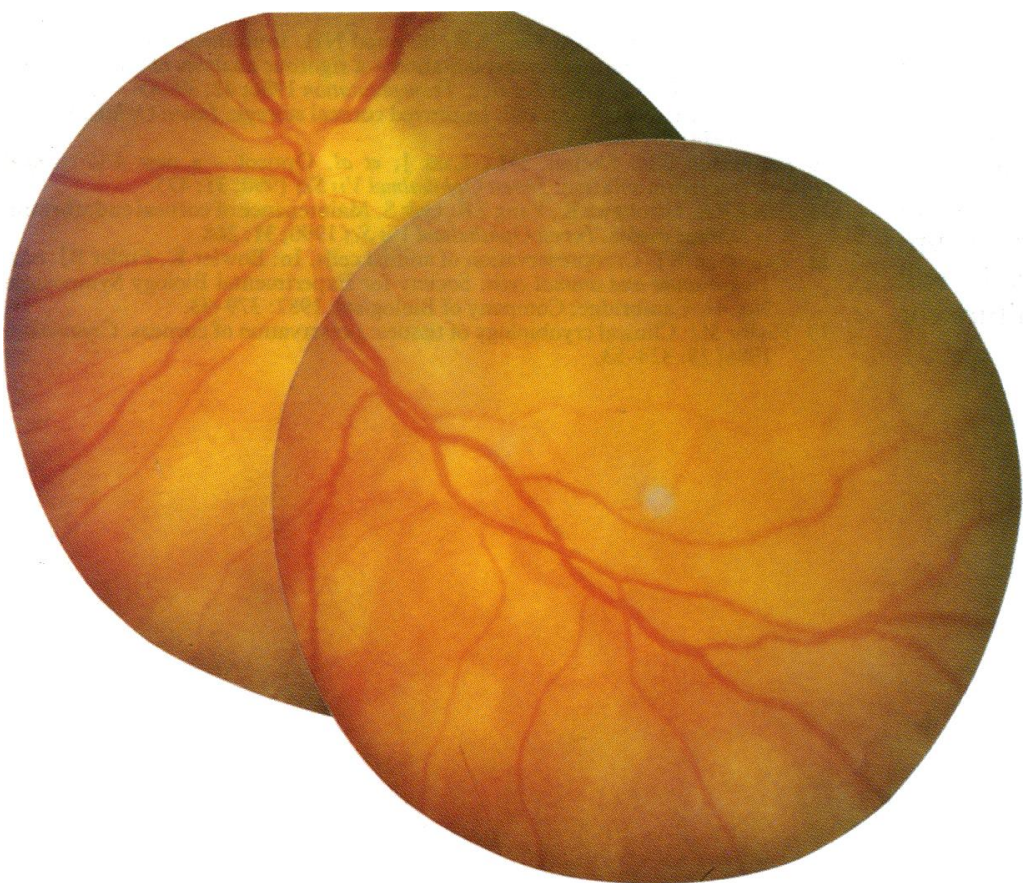

Figure 1 The appearance of the left fundus at the time of presentation in 1978. Note the multiple pale lesions scattered across the central retina. material has in many cases prevented an understanding of the aetiology of these conditions. In this report we describe the unexpectedly active and destructive inflammatory changes found in a case of multifocal choroiditis which clinically was thought to be quiescent.

\section{Case report}

In 1978 a 50 -year-old woman presented to the eye casualty department with a one-month history of aching pain and blurring vision in both eyes. Her vision was $6 / 12$ in the right and $6 / 18$ in the left eye. She had mild iritis and mild optic disc oedema in both eyes. Two weeks later her vision deteriorated to counting fingers right and hand movements left. In addition to moderate anterior chamber inflammatory activity she had bilateral total exudative retinal detachments. She had no systemic illness, was on no medication, and extensive investigation including viral serology gave negative results.

Treatment with systemic prednisolone $80 \mathrm{mg} /$ day resulted in rapid regression of the exudative detachments; cream coloured round patches then became evident throughout the whole extent of both fundi (Fig 1). Fluorescein angiography showed patchy late hyperfluorescence. The electro-oculogram (EOG) was reduced to $140 \%$ in the right eye and $120 \%$ in the left. A provisional diagnosis of acute posterior multifocal placoid pigment epitheliopathy (APMPPE) was made. The patient's vision gradually improved, and the steroid dose was reduced, though two transient episodes of increased activity required temporary increases in steroid dosage. One year after presentation her visual acuity was $6 / 9$ right and $6 / 6$ left, and there was patchy depigmentary chorioretinal scarring, with mottled pigmentation of the maculae (Fig 2).

Low dose systemic steroids were continued for a total of five years. There was mild residual anterior uveitis following systemic steroid withdrawal. The patient died in 1987 from an acute myocardial infarct. Six weeks prior to death her vision was $6 / 9$ right and left, there was a mild bilateral anterior uveitis, and there were patches of chorioretinal scarring which did not show any clinical evidence of active inflammation.

Material and methods

The eyes were removed approximately 12 hours after death. The left eye was fixed in formal 


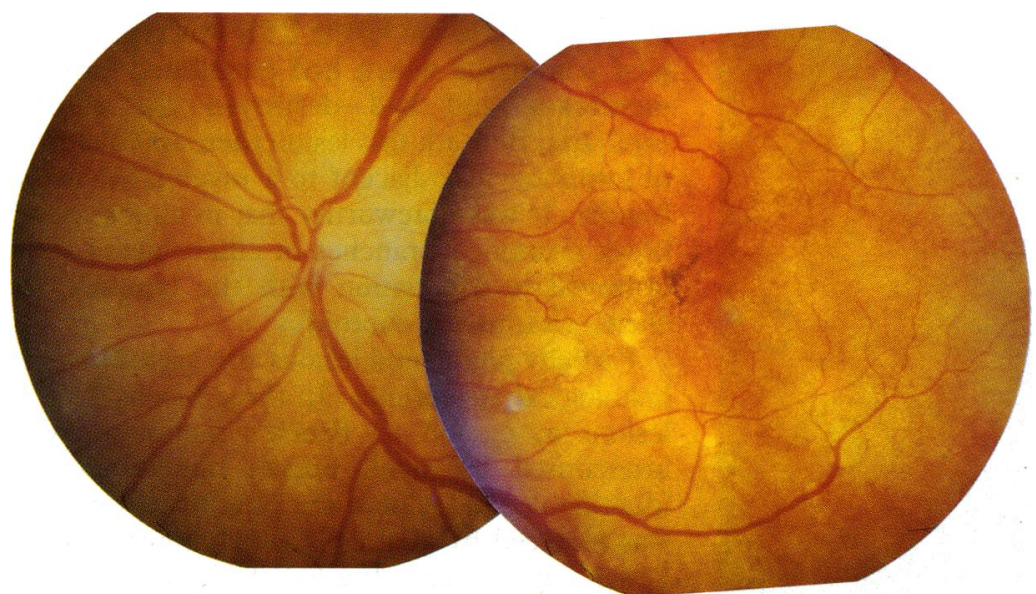

Figure 2 Left fundal appearance one year later. Note the hyperpigmentation at the macula and the loss of pigmentation elsewhere.

saline and the right in $2 \%$ cacodylate buffered glutaraldehyde. Both eyes were of normal dimensions and were divided horizontally above and below the macula.

Paraffin-histology. After paraffin embedding serial sections were taken from both paraffin blocks and stained with haematoxylin-eosin (H-E), periodic acid Schiff (PAS), and stains for axons (Bodain), myelin (Loyez), and iron (Perls). Additional blocks were taken from the calottes for immunohistochemistry and electron microscopy.

Immunohistochemistry. Sections from the formal saline fixed tissue were stained by the peroxidase-antiperoxidase (PAP) technique for $B$ and $T$ lymphocytes, macrophages (muraminidase, $\alpha$-ACT, $\alpha-1 \mathrm{AT}$ ), and immunoglobulins (IgA, IgG, IgM, $x$ and $\lambda$ ). In-situ hybridisation was employed for identification of herpes simplex viral antigen.

Electron microscopy. Macrophotographs were taken of the superior and inferior calottes from the right eye, and designated foci of hypo and hyperpigmentation were excised for study (Figs 3 and 4). Fifteen blocks were embedded in Araldite and $1 \mu \mathrm{m}$ sections were stained with toluidene blue. Eight blocks were studied by

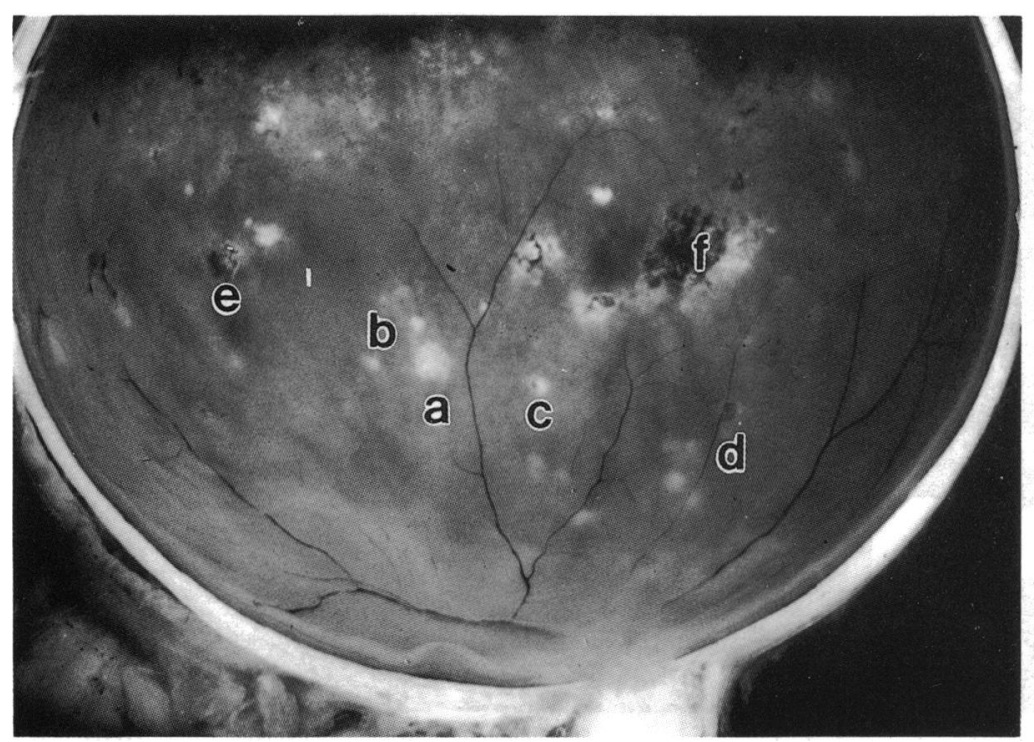

Figure 3 Inferior calotte of the right eye to show the macroscopic appearance of the lesions. $E M$ blocks were taken from areas designated a-f (letters located below lesions). semiserial sectioning (at 50 or $100 \mu \mathrm{m}$ steps), and, where appropriate, ultrathin sections were taken and stained conventionally for examination in a Philips 301 electron microscope.

\section{Results}

MACROSCOPIC EXAMINATION

The findings in both globes were essentially the same. The anterior segments appeared normal, and the vitreous was clear. There were meridional folds on the pars plana, but no evidence of pars planitis. Focal lesions, varying in size between 0.5 and $1.5 \mathrm{~mm}$, were scattered throughout the fundus, predominantly in the mid periphery. The smallest lesions were pale and free from pigmentation, while the more obvious contained specks and clumps of pigment (Figs 3 and 4). The largest lesions were heavily pigmented with lacunae of depigmentation. The appearances of the maculae, the peripapillary retinae, and the optic discs were within normal limits for postmortem material.

\section{MICROSCOPIC EXAMINATION \\ Paraffin sections}

The conjunctiva, the cornea, the outflow system, and the lens were of normal architecture. The iris stroma contained scattered lymphocytes which formed aggregates at the pupil and at the root. The ciliary body contained a few clusters of lymphocytes, and there were non-specific proliferative changes in the epithelial layers of the pars plana. The vitreous was free from inflammatory cell infiltration.

At the far periphery the retinal pigment had non-specific age-related degenerative changes, and occasional drusen were observed. The inner retina was well preserved, and the nerve fibre layer and optic nerve contained axons of normal density.

At the macula there was depletion of nuclei in the outer nuclear layer; here the photoreceptors showed autolytic changes. Clusters of lymphocytes were noted in the posterior choroid and in the adventitia of some of the posterior ciliary vessels and nerves. A study of designated representative lesions (Figs 3 and 4) was made from plastic embedded matrial.

\section{Plastic embedded histology}

The following description is derived from blocks taken from the sites designated in Figs 3 and 4. Where appropriate light microscopy was supplemented by electron microscopy. The descriptions relate to the centre of the lesions.

The small non-pigmented lesions were of variable histological appearance. In three blocks (Fig 4, lesions P, T, and W) there was a lymphocytic infiltration in the choroid, with some cellular infiltration in the hypopigmented retinal pigment epithelium (Fig 5); the overlying retina showed minor atrophy of the photoreceptor layer. Electron microscopy showed the cells within the retinal pigment epithelium (RPE) to be macrophages (Fig 5C) and the outer segments to be adequately preserved (Fig 5 b). By contrast in a somewhat similar macroscopic 


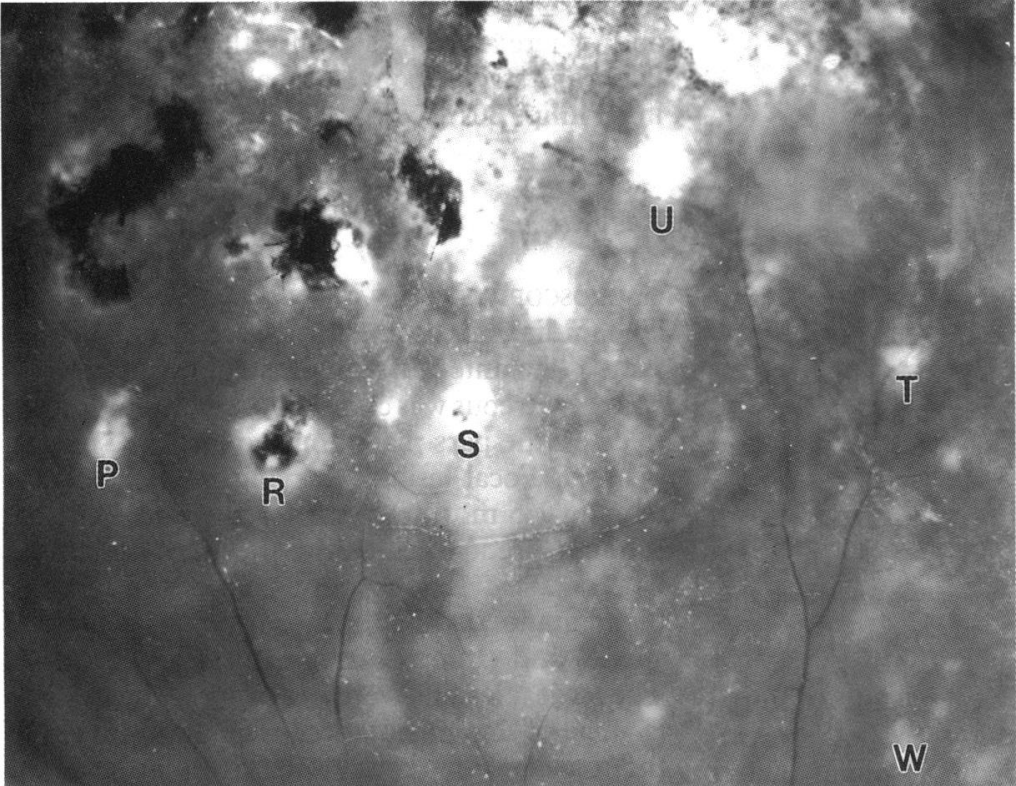

lesion (Fig 3, lesion b) the RPE was replaced by glial scar tissue which had penetrated Bruch's membrane and had destroyed the choriocapillaris. Beneath this lesion there was a cluster of lymphocytes adjacent to a myelinated nerve bundle. It was noteworthy that in almost every block examined branches of ciliary nerve were observed in association with the lymphocytic clusters within the choroid.

Partially pigmented lesions also varied in their histological appearance. In three blocks (Fig 3, lesions a, c, and d) Bruch's membrane was interrupted by small breaks but was easily identifiable. In lesion $c$ the photoreceptor layer was replaced by glial cells which abutted on to Bruch's membrane (Fig 6a). In lesions a and d (Fig 3) the RPE was multilayered and infiltrated by macrophages and lymphocytes (Figs $6 \mathrm{~b}, \mathrm{c}$ ). Over these areas of RPE disturbance there was outer retinal atrophy of varying degrees. The choriocapillaris was either normal, infiltrated by inflammatory cells, or replaced by collagenous

Figure 4 The appearance of the superior calotte to show in more detail the characteristic lesions. EM blocks were taken from the areas designated $P-W$.
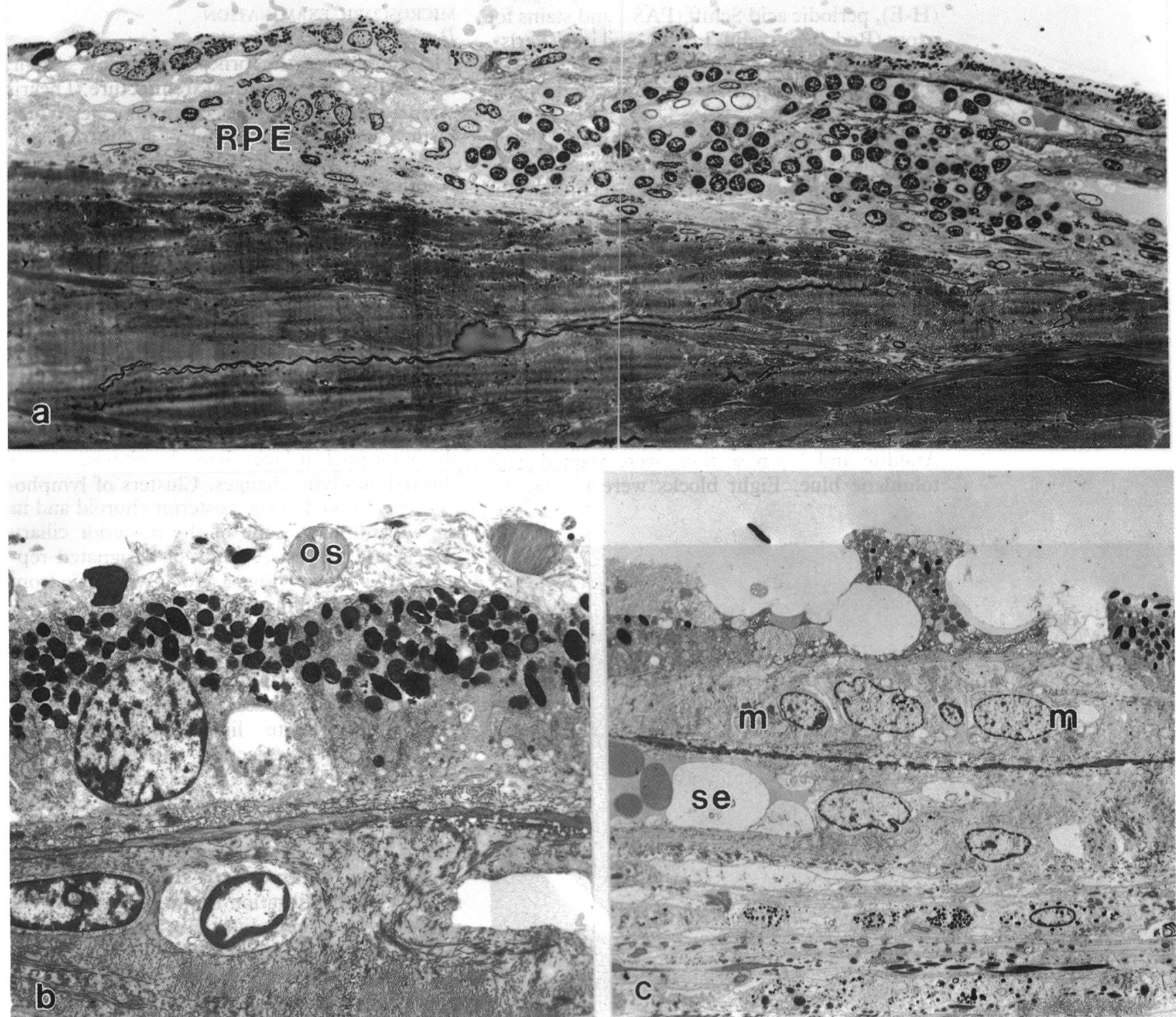

Figure 5 The choroid in small pale lesions. (a) The stroma contains lymphocytes (which are also present in the choriocapillaris) and RPE clusters (RPE). (b) Outer segments $(o s)$ are present on the surface of the RPE. (c) Macrophages $(m)$ are infiltrating the pigment epithelium. The choriocapillaris is partially replaced by fibrous tissue and elsewhere the endothelial cells are swollen (se). $(a, \times 250 . b, \times 3000 . c, \times 1700$. 

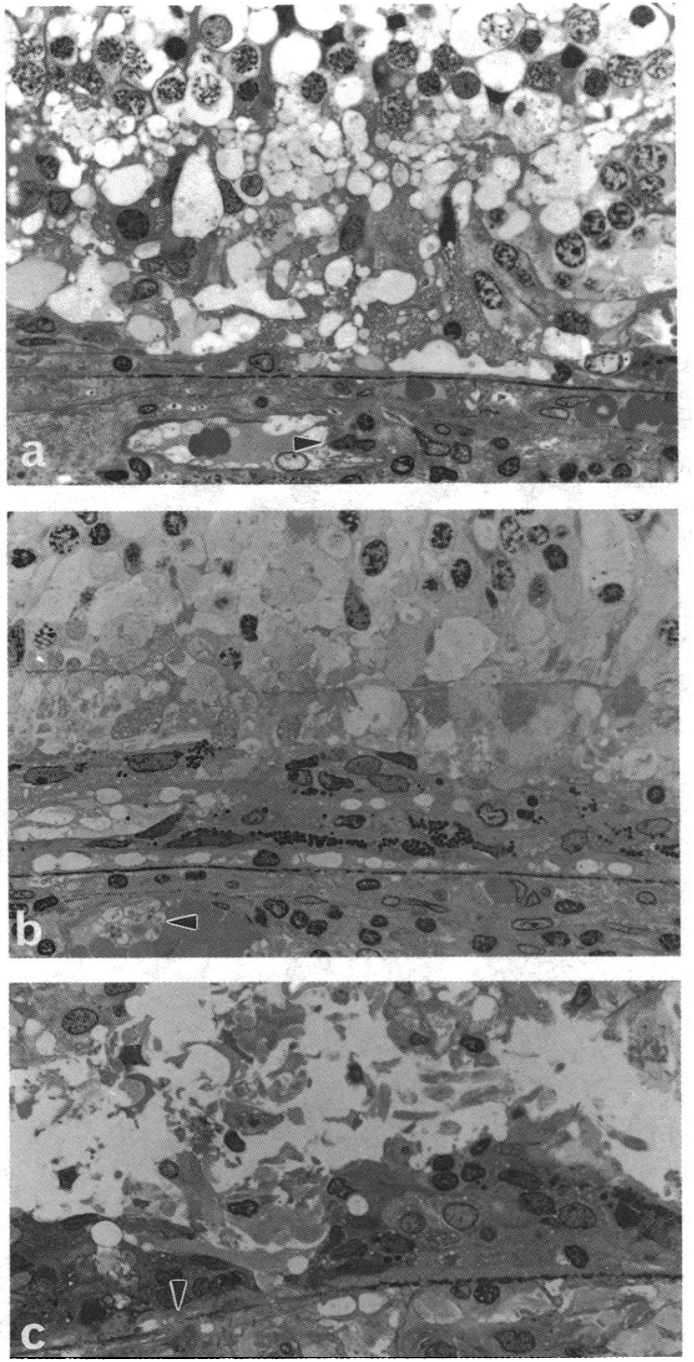

Figure 6 Light micrographs of lesions c (top), d (middle), and $a$ (below) shown in figure 3. Bruch's membrane is intact in all three examples. (a) Inflammatory cells are sparse and gliotic retina abuts on to the membrane. (b) There is multilayering of the RPE but there is some preservation of the inner segments. (c) The photoreceptors are fragmented over clusters of RPE cells, macrophages, and lymphocytes. Note inflammatory cells in swollen endothelium in $(a)$, a platelet aggregate in (b), and a macrophage within Bruch's membrane in (c) (arrow heads). $(\times 375$.

tissue. Invariably there were clusters of lymphocytes in the choroidal stroma.

In other partially pigmented lesions the inflammatory process was more destructive (Fig 3, lesion e, Fig 4, lesion s). In these it was difficult to identify Bruch's membrane. The outer retina was invaded by lymphocytes, macrophages, RPE cells, and capillaries (Fig 7).

Swelling and cytoplasmic rarefaction of endothelial cells in choroidal capillaries and venules was a constant feature irrespective of the degree of inflammatory destruction (Figs 5, 6a, and 7). At the ultrastructural level the junctions between these endothelial cells were intact, but lymphocytes were often found in close relationship with the endothelial cells (Fig 6a). In some vascular lumina there were platelet clusters (Fig 6b), but neither fibrin, thrombi, nor immune complex deposits were identified. There was no evidence of previous choroidal vascular occlusive disease as shown by hyalinisation of the intima or media. Migration of RPE cells into the choroid was seen in lesions of varied appearance and degree of destruction (Figs 5 and 7).

Two heavily pigmented foci were studied (Fig 3, lesion $\mathbf{f}$, and Fig 4, lesion R). These consisted of atrophic retina in all layers over a band of multilayered and hyperplastic RPE cells with an admixture of macrophages. There was a dense choroidal inflammatory infiltrate. At the periphery of even advanced lesions the photoreceptor outer segment architecture was preserved, and evidence of photoreceptor phagocytosis by exogenous macrophages was obtained (Fig 8).

In none of the sections examined at the light or ultrastructural level was it possible to identify structures with protozoal, viral, or bacterial characteristics.

\section{Immunohistochemistry}

Within the choroidal infiltrate $70-80 \%$ of the lymphocytes were identified as $T$ cells, less than $20 \%$ were B cells. Small numbers of macrophages were noted in all parts of the inflammatory foci. There was no significant deposition of immunoglobulin. In-situ hybridisation for identification of herpes simplex virus gave negative results.

\section{Discussion}

The case was initially diagnosed as acute posterior multifocal placoid pigment epitheliopathy (APMPPE)' because of the multiple pale inflammatory lesions seen across the posterior fundi. Exudative retinal detachments have been described as an unusual presenting feature of APMPPE, ${ }^{2}$ and more recent reports have stressed that APMPPE can be chronic or recurrent. ${ }^{3-5}$ However, the presentation and subsequent course of the disease are more typical of Harada's disease. ${ }^{6-8}$ Similarities between these two conditions have been noted, and it has been suggested that they represent a continuous spectrum of disease. ${ }^{9}$ This case has the ocular picture of Harada's disease without the systemic manifestations and therefore best fits an intermediate group as defined by Wright, Bird, and Hamilton.' Because of the difficulties in categorising the case we have elected to refer to it in the broader clinical context of multifocal posterior uveitis.

The histopathological characteristics of the lesions identified in the choroid and retina include a deep choroidal lymphocytic infiltrate (usually associated with a myelinated nerve), with extension to the choriocapillaris and variable breaks in Bruch's membrane. Thickening of the RPE was due to proliferation of the native RPE cells (with and without melanin depletion) and to infiltration by macrophages and lymphocytes. The restricted focal nature of the lesions was impressive, as was the sparing of the adjacent retina. Indeed it appeared that involvement of the photoreceptor layer could be regarded as the destruction of an innocent bystander. The pattern of cellular infiltration is similar to that found by Perry and Font in cases of Harada's disease, ${ }^{7}$ although we found few plasma cells within the infiltrate.

It has been suggested that both APMPPE and multifocal choroiditis may be initiated by choroidal ischaemia. ${ }^{9-13}$ With reference to this 

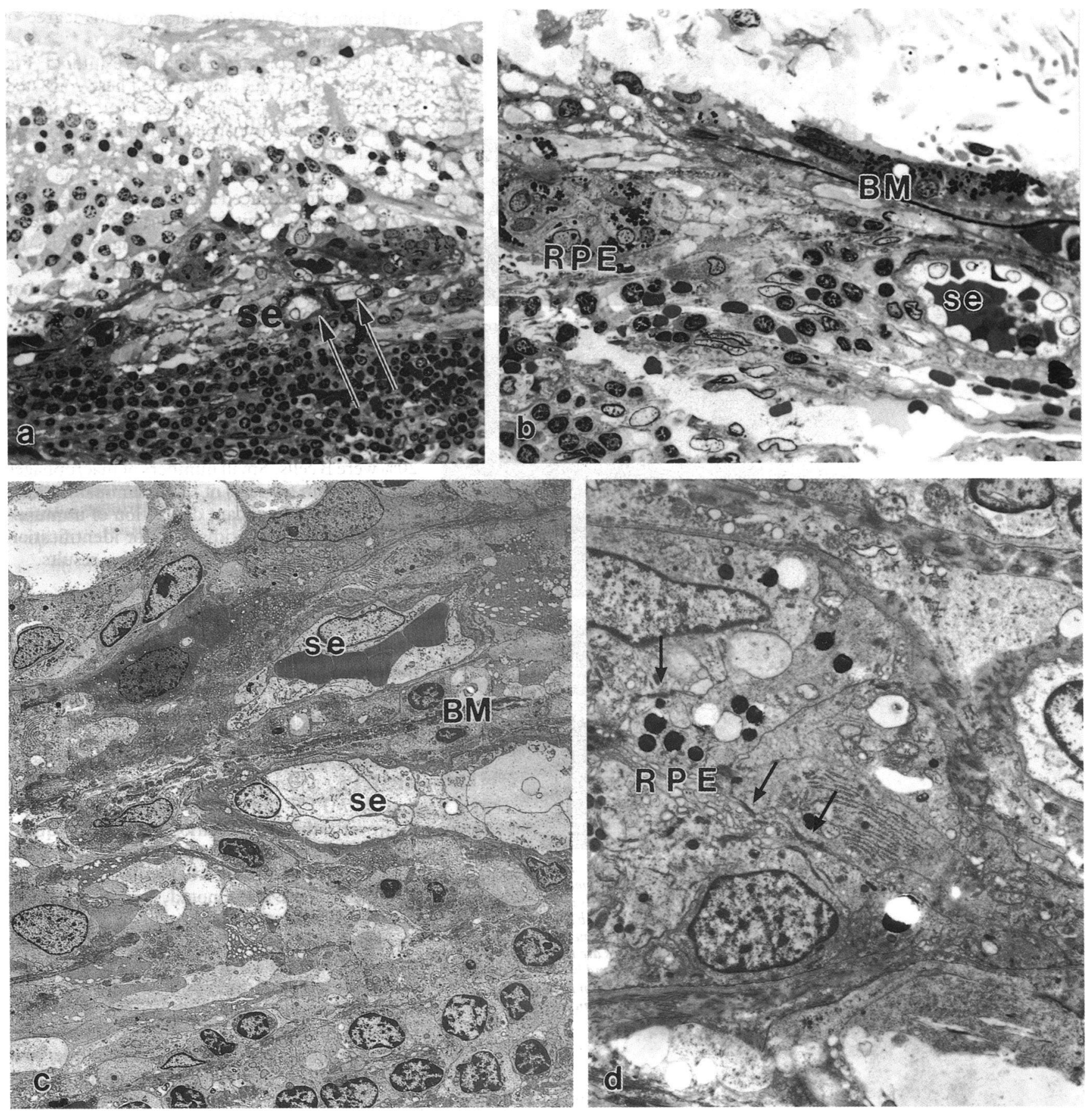

Figure 7 Lesion $S$ in Fig 4. The inflammatory reaction had penetrated Bruch's membrane. (a) The outer retina is destroyed by an infiltrate which contains lymphocytes, macrophages, and capillaries (arrows), with swollen endothelial cells (se). (b) The choroid adjacent to the focus contains $R P E$ cells $(R P E)$, and the venule is lined by swollen endothelial cells (se). The capillaries present on both sides of Bruch's membrane are shown in more detail in $(c)$. (d) This demonstrates the junctional attachments between $R P E$ cells $(R P E)$ within the choroid (arrows). $(a, \times 250 . b, \times 630 . c, \times 1500 . d, \times 4300$.)

hypothesis we did not find immune complexes or fibrinoid necrosis in blood vessels, nor was there any evidence to suggest a previous inflammatory vasculopathy which had produced choroidal vascular hyalinisation and luminal narrowing. There was no evidence of current bacterial, fungal, viral, or protozoal infection.

The evolution of the destructive process appeared to depend on the preservation of Bruch's membrane. If the membrane survived the initial inflammatory insult, the RPE responded by proliferation and formation of layers of collagen. An intact Bruch's membrane did not necessarily protect the RPE and photoreceptor layer. RPE loss and photoreceptor destruction were seen despite the preservation of the under- lying membrane, and we demonstrated that inflammatory cells may gain access to the outer retina through small breaks in an otherwise intact Bruch's membrane (Fig 6).

There was notable photoreceptor survival despite ongoing inflammation within the foci. The long term survival of photoreceptors contrasts with the early lysis of rod outer segments reported in experimental autoimmune uveitis. ${ }^{14}$ Our evidence suggests that photoreceptor outer segments are not the primary autoimmune target in this form of human disease.

Glial cell migration from the retina to the choroid has been described in syphilis ${ }^{15}$ and other inflammatory conditions. ${ }^{16}$ The occurrence of this abnormality in multifocal posterior uveitis 


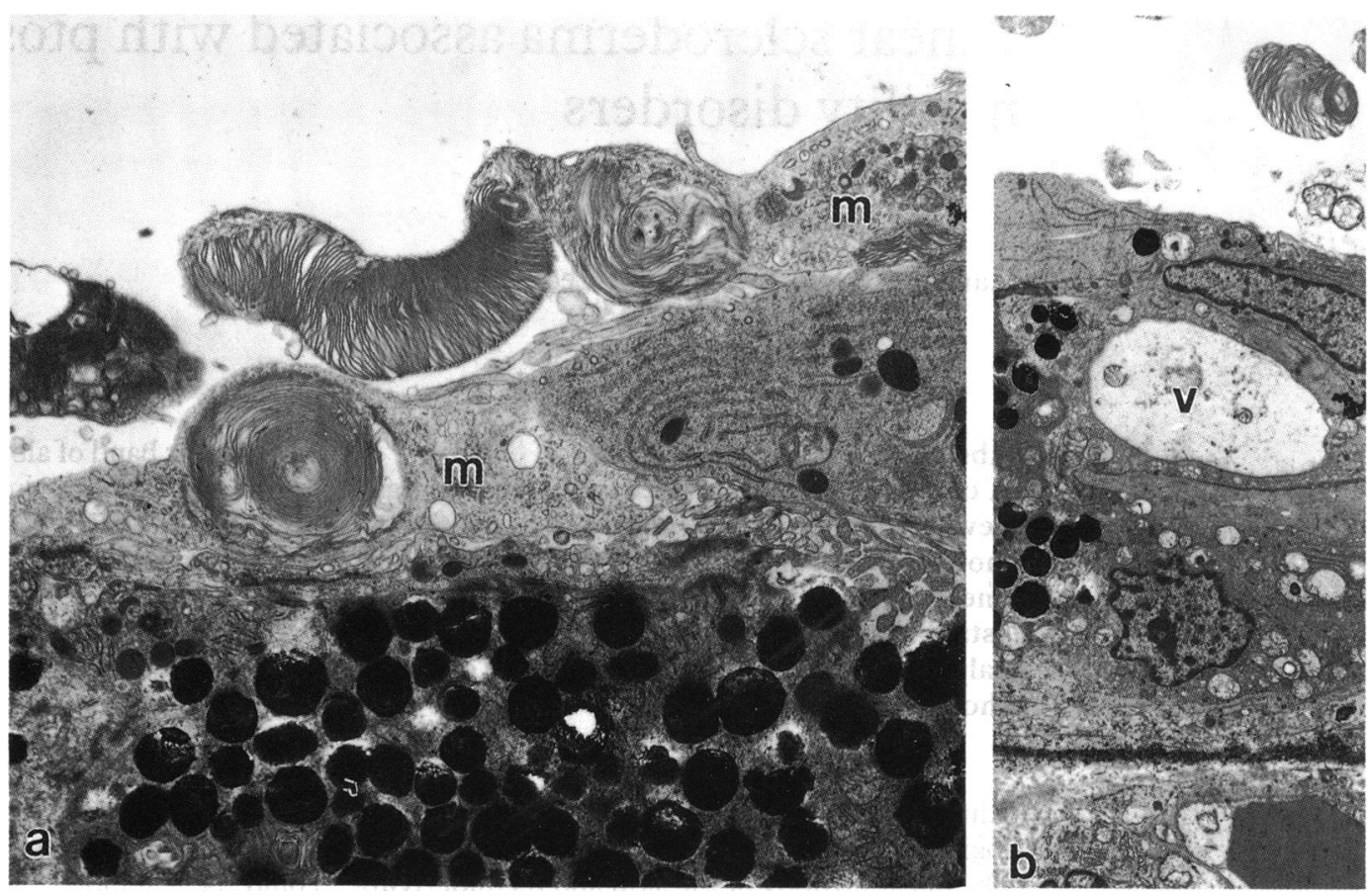

Figure 8 Electron micrographs to illustrate the preservation of the outer segments of the photoreceptors, which in this example are phagocytosed by macrophages $(m)$. Some $R P E$ cells contain vacuoles $(v) .(a, \times 6000 . b, \times 2480$.)

is further evidence of the non-specific nature of the glial cell activity. Similarly migration of RPE cells into the choroid is now regarded as nonspecific, ${ }^{16}$ as is neovascularisation in a glial scar. ${ }^{17}$

Recent work has highlighted the importance of high endothelial venule (HEV) like vessels in the recruitment of lymphocytes into inflamed tissues. ${ }^{1819}$ The term 'high endothelial venule' is applied to the post-capillary venules of lymph nodes and to vessels in inflamed tissue which morphologically have an increase in the height of their endothelial cells. These endothelial cells have histochemical alterations relating to lymphocyte-endothelial interactions and are believed to play a role in the uptake of lymphocytes to lymphatic and inflamed tissues. We frequently found this response to dense focal accumulations of lymphocytes, and we also demonstrated the presence of lymphocytes within the vessel wall. Endothelial cell swelling was demonstrated in both the choroidal venules and the choriocapillaris. The presence of such changes is of added significance in view of the recently reported association of class II major histocompatibility complex (MHC) antigen expression on endothelial cells with lymphocyte recruitment in experimental ocular inflammation..$^{20}$

In conclusion, we have demonstrated that active, ongoing focal inflammation can occur in a case of clinically quiescent posterior uveitis. Despite detailed study we were unable to correlate the macroscopic appearance of the lesions with specific patterns of pathological change. No ischaemic or infective cause of the disorder was found.

There was migration of glial and RPE cells within the inflammatory foci and neovascularisation of the glial scars; these changes are seen in other forms of posterior uveitis. We believe that this is the first study to demonstrate the presence of high endothelial venule-like vessels at the sites of inflammation in human ocular tissue.

1 Gass JDM. Acute posterior multifocal placoid pigment epitheliopathy. Arch Ophthalmol 1968; 80: 177-85.

2 Bird AC, Hamilton AM. Placoid pigment epitheliopathy presenting with bilateral serous retinal detachment. $B r \mathcal{F}$ Ophthalmol 1972; 56: 881-6.

3 Murray SB. Acute posterior multifocal placoid pigment epitheliopathy: Not so benign? Trans Ophthalmol Soc UK 1979; 99: 497-500.

4 Damato BE, Nanjiani M, Foulds WS. Acute posterior multifocal placoid pigment epitheliopathy. A follow up study. Trans Ophthalmol Soc UK 1983; 103: 517-22.

5 Gass JDM. Acute posterior multifocal placoid pigment epitheliopathy. A long term follow up. In: Fine SL, Owens $\mathrm{L}$, eds. Management of retinal vascular and macular disorders. Baltimore: Williams and Wilkins, 1983: 176-81.

6 Ohno S, Char DH, Kimura SJ, O'Connor GR. VogtKoyanaga-Harada syndrome. Am f Ophthalmol 1977; 83: $735-40$.

7 Perry HD, Font RL. Clinical and histopathological observations in severe Vogt-Koyanagi-Harada syndrome. Am $\mathcal{J}$ vathalmol 1977; 83: 242-54.

8 Chan C, Palestine A, Kuwabara L, Nussenblatt R. Immunopathological study of Vogt-Koyanagi-Harada syndrome. Am F Ophthalmol 1988; 105: 607-11.

9 Wright BE, Bird AC, Hamilton AM. Placoid pigment epitheliopathy and Harada's disease. Br $\mathcal{F}$ Ophthalmol 1978; 62: 609-21.

10 Hayreh SS. Segmental nature of the choroidal vasculature. Brf Ophthalmol 1975; 59: 631-47.

11 Deutman AF, Lion F. Choriocapillaris nonperfusion in acute multifocal placoid pigment epitheliopathy. Am $\mathcal{F}$ Ophthalmol 1977; 83: 652-7.

12 Young NJA, Bird AC, Sehmi K. Pigment epithelial diseases with abnormal choroidal perfusion. Am $\mathcal{f}$ Ophthalmol 1980; 90: 607-17.

13 Gaudric A, Coscas G, Bird AC. Choroidal ischemia. Am $\mathcal{J}$ Ophthalmol 1982; 94: 489-98.

14 Forrester JV, Borthwick GM, McMenamin PG. Ultrastructural pathology of S-antigen uveoretinitis. Invest Ophthalmol

15 Blodi FC, Hervovet F. Syphilitic chorioretinitis. Arch Ophthalmol 1968; 79: 294-6.

16 Green WR. Retina. In: Spencer WH, ed. Ophthalmic pathology. Philadelphia: Saunders, 1985: 855.

17 Green WR. Uveal tract. In: Spencer WH, ed. Ophthalmic pathology. Philadelphia: Saunders, 1986; 1513-4.

18 Freemont AJ, Ford WL. Functional and morphological changes in post-capillary venules in relation to lymphocytic infiltration into BCG-induced granulomata in rat skin. f Pathol 1985; 147: 1-12.

19 Freemont AJ. Functional and biosynthetic changes in endothelial cells of vessels in chronically inflamed tissues: evidence for endothelial control of lymphocyte entry into diseased tissues. F Pathol 1988; 155: 225-30.

20 Lightman S. Immune mechanisms in autoimmune ocular disease. Eye 1988; 2: 260-6. 